\title{
Hypertriglyceridemia-Induced Pancreatitis in Poorly Controlled Type 2 Diabetes
}

\section{Hyun-Ho Jo, Kyu-Jin Kim, Bo-Yeon Kim, Chan-Hee Jung, Chul-Hee Kim, Sung-Koo Kang, Ji-Oh Mok}

Division of Endocrinology \& Metabolism, Department of Internal Medicine, Soonchunhyang University Bucheon Hospital, Soonchunhyang University College of Medicine, Bucheon, Korea

\begin{abstract}
A 38-year-old female presented with abdominal pain, radiating to her back. Her medical history included type 2 diabetes, which had been uncontrolled for 8 months. Her initial laboratory tests showed marked hyperglycemia, metabolic acidosis, and elevated serum amylase and lipase levels, although the results were inconclusive in terms of a direct diagnosis of acute pancreatitis (AP). Abdominal computed tomography showed only minimal fluid collection at the pancreas tail. As her serum triglyceride (TG) level was 9,884 mg/dL, we made a working diagnosis of AP due to hypertriglyceridemia, and she was treated with massive hydration with an insulin infusion. Subsequently, she recovered rapidly from the abdominal pain, her serum glucose was controlled, and her serum TG decreased. Hypertriglyceridemia is a well-accepted underlying cause of AP. When extremely high hypertriglyceridemia is detected in patients with type 2 diabetes or metabolic syndrome, complications should be considered and managed.
\end{abstract}

Keywords: Pancreatitis; Hypertriglyceridemia; Diabetes mellitus, type 2

\section{INTRODUCTION}

Acute pancreatitis (AP) is a common condition with various etiologies, most commonly gall stones and alcohol [1]. Although less frequent, hypertriglyceridemia causes about $10 \%$ of all AP cases [2], the hypertriglyceridemia is primary in less than $5 \%$ of the cases, due to genetic causes, and more often secondary to other causes, such as type 2 diabetes, obesity, pregnancy, excess carbohydrate intake, hypothyroidism, alcohol, hepatitis, sepsis, renal failure, and drugs such as estrogen, glucocorticoids, $\beta$-blockers, bile acid binding resins, thiazides, tamoxifen, cyclosporine protease inhibitors, and isotretinoin [3]. A serum triglycerides (TG) level exceeding $1,000 \mathrm{mg} / \mathrm{dL}$ increases the likelihood of AP [4]. Here, we report the case of a female with poorly controlled type 2 diabetes presenting with recurrent pancreatitis, with features of hypertriglyceridemia.

\section{CASE REPORT}

A 38-year-old female presented to the Emergency Department with chief complaint of epigastric pain, radiating to the back, for 3 days. She was a non-smoker and non-drinker. She did not take herbal medicines or folk remedies. Her defecation pattern was normal. She had no history of trauma or abdominal surgery. She had no recent history of taking other medications, such as oral contraceptives or anti-hypertensives. Her father had type 2 diabetes, but she had no family history of premature cardiovascular disease or dyslipidemia. As her father, she was also a diabetic. She was diagnosed with gestational diabetes mellitus, and treated with insulin injection eight years earlier. One year after her delivery, her serum glucose was checked; it was found to be as high as $400 \mathrm{mg} /$ $\mathrm{dL}$, and she continued on oral hypoglycemic agents. But she had not been paying attention to her medication, had poorly controlled serum glucose, and did not visit the clinic regularly. She had been admitted to hospital for AP on five previous occasions.

In this visit, she was alert, and the initial vital signs were blood pressure $120 / 80 \mathrm{~mm} \mathrm{Hg}$, pulse 80 beats/min, respiratory rate 18/ min, and body temperature $36.5^{\circ} \mathrm{C}$. She weighed $50.8 \mathrm{~kg}$ and was $154 \mathrm{~cm}$ in height, for a body mass index of $21.42 \mathrm{~kg} / \mathrm{m}^{2}$. Her ini-
Correspondence to: Ji-Oh Mok

Division of Endocrinology \& Metabolism, Department of Internal Medicine, Soonchunhyang University Bucheon Hospital, Soonchunhyang University College of Medicine, 170 Jomaru-ro, Wonmi-gu, Bucheon 420-767, Korea

Tel: +82-32-621-5156, Fax: +82-32-621-5018, E-mail: hanna@schmc.ac.kr

Received: Sep. 12, 2014 / Accepted after revision: Nov. 17, 2014 (c) 2014 Soonchunhyang Medical Research Institute This is an Open Access article distributed under the terms of the Creative Commons Attribution Non-Commercial License (http://creativecommons.org/licenses/by-nc/3.0/). 
Table 1. Initial laboratory parameters

\begin{tabular}{|c|c|c|}
\hline Variable & Value & Reference range \\
\hline White blood cell $\left(\times 10^{3} / \mu \mathrm{L}\right)$ & 8.75 & $4,000-10,000$ \\
\hline Hemoglobin (g/dL) & 15.0 & $12-16$ \\
\hline Platelet count $\left(\times 10^{3} / \mu \mathrm{L}\right)$ & 331 & $150-450$ \\
\hline Glucose (mg/dL) & 397 & $60-108$ \\
\hline Blood urea nitrogen (mg/dL) & 9.9 & $8-20$ \\
\hline Creatinine (mg/dL) & 0.7 & $0.6-1.3$ \\
\hline Sodium (mEq/L) & 130 & $135-145$ \\
\hline Potassium (mEq/L) & 4.2 & $3.5-5.5$ \\
\hline Chloride (mEq/L) & 98 & $98-110$ \\
\hline Aspartate aminotransferase (IU/L) & 12 & $5-40$ \\
\hline Alanine aminotransferase (IU/L) & 10 & $0-40$ \\
\hline C-reactive protein (mg/L) & 0.7 & $0-0.5$ \\
\hline Amylase (U/L) & 205 & $28-100$ \\
\hline Lipase (IU/L) & 398 & $7-60$ \\
\hline Hemoglobin A1C (\%) & 14.0 & $4-6$ \\
\hline Thyroid stimulating hormone $(\mu \mathrm{U} \mathrm{U} / \mathrm{mL})$ & 0.58 & $0.39-5.44$ \\
\hline Free T4 (ng/dL) & 1.17 & $0.8-2$ \\
\hline Total cholesterol (mg/dL) & 878 & $90-250$ \\
\hline Triglyceride (mg/dL) & 9,880 & $0-200$ \\
\hline Low density lipoprotein cholesterol (mg/dL) & 316 & $0-140$ \\
\hline High density lipoprotein cholesterol (mg/dL) & 28 & $45-65$ \\
\hline Apoprotein A1 (mg/dL) & 118 & $102-181$ \\
\hline Apolipoprotein B (mg/dL) & 159 & $51-123$ \\
\hline Basal C-peptide (ng/mL) & 1.55 & $0.48-3.30$ \\
\hline 120-minute postprandial C-peptide (ng/mL) & 2.67 & $4,000-10,000$ \\
\hline $\begin{array}{l}\text { Homeostasis model assessment insulin re- } \\
\text { sistance }\end{array}$ & 2.37 & $12-16$ \\
\hline
\end{tabular}

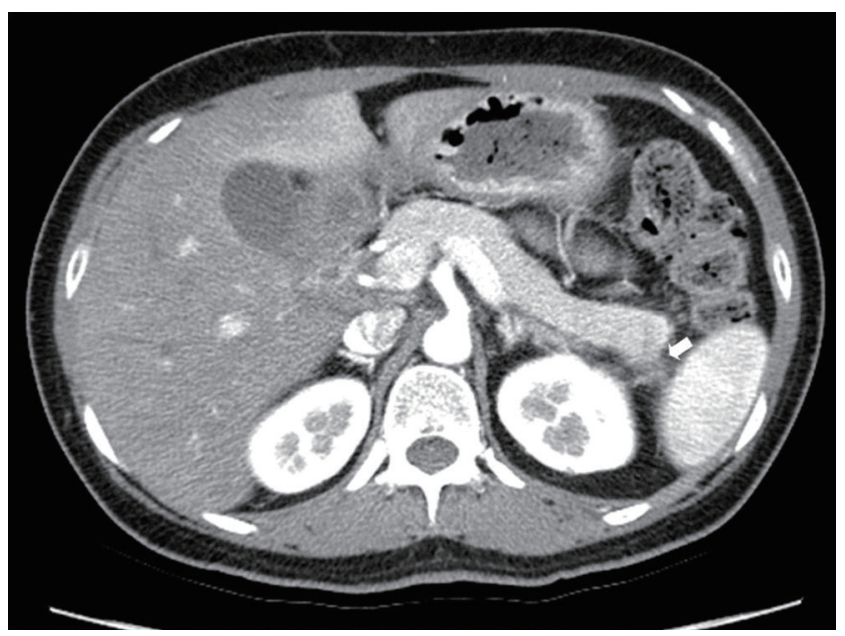

Fig. 1. Abdominal computed tomography image shows a pancreas with focal fat in filtration in the pancreatic tail (white arrow).

tial laboratory parameters are shown in Table 1. Her physical examination showed no xanthelasmas or skin eruptions, nor was a fruity odor detected. She was admitted to the Gastroenterology
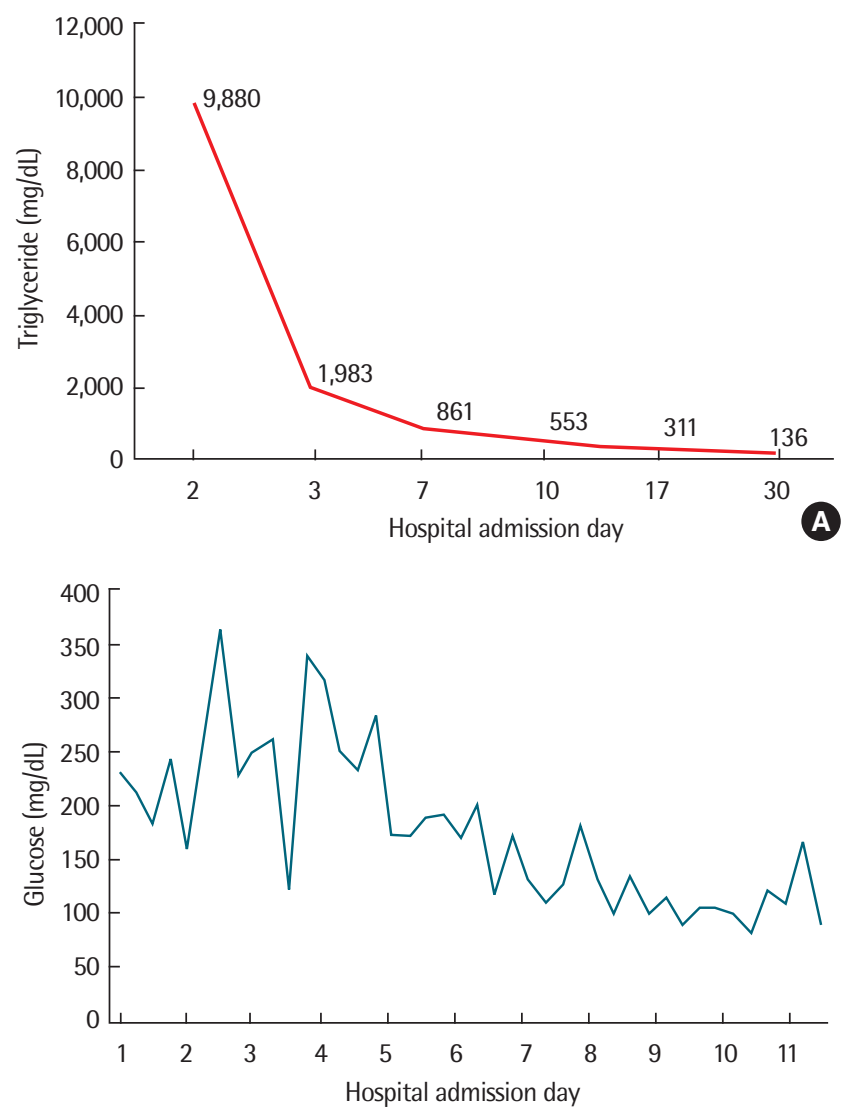

\begin{tabular}{|l|l|l|l|}
\hline Insulin & Infusion & Multiple injection \\
\hline \hline Diet & NPO & Diabetic low fat diet $(1,500 \mathrm{kcal})$ \\
\hline
\end{tabular}

Fig. 2. (A) Serum triglyceride levels. (B) Serum glucose levels during the admission. Insulin intravenous infusion was done until hospital admission day 3, followed by insulin multiple injection. Serum triglyceride dropped to normal range within 12 days. The patient started on 1,500-calorie low fat diabetic diet on day 2.

Department for abdominal pain, treated with intravenous saline infusion, and allowed nothing by mouth. The pain subsided. After the initial hydration, computed tomography (CT) was performed within 48 hours owing to her episodes of recurrent pancreatitis and epigastric pain. This showed segmental adenomyomatosis of the gallbladder and focal duct dilatation in pancreatic tail, suggestive of combined focal pancreatitis. CT images are shown in Fig. 1. A diagnosis of AP due to very high TG levels was made. Treatment with intravenous fluids mixed with insulin was started. Her serum glucose decreased from $397 \mathrm{mg} / \mathrm{dL}$ at admission to $233 \mathrm{mg} / \mathrm{dL}$ the night of admission (Fig. 2). She was started on a 1,500-calorie low fat diabetic diet on day 2 , continued her insulin mixed fluid infusion, and was transferred to Endocrinology Department to control her type 2 diabetes and hypertriglyceridemia on day 3 . There, she was started on insulin glargine 20 
IU at bedtime and insulin lispro $8 \mathrm{IU}$ before each meal. Her serum TG decreased to $1,983 \mathrm{mg} / \mathrm{dL}$ on day 3 and to $861 \mathrm{mg} / \mathrm{dL}$ on day 6 . On day 12, she was discharged on insulin glargine 35 IU once at bedtime, insulin lispro 3 IU before each meal, metformin $500 \mathrm{mg}$ bid, rosuvastatin $20 \mathrm{mg}$ qd, and fenofibrate $160 \mathrm{mg}$ qd. She remained asymptomatic with normal TG levels at the 1-month follow-up. The presumed cause of the hypertriglyceridemia was uncontrolled type 2 diabetes.

\section{DISCUSSION}

Hypertriglyceridemia is interrelated with serum glucose and insulin resistance. Type 2 diabetes alters lipid metabolism, which produces an increase in lipoprotein production, while reducing the rate of lipoprotein clearance [5]. Visceral adiposity with insulin resistance is resistant to the anti-lipolytic action of insulin, leading to a hyperlipolytic state [6,7]. This results in increased intracellular hydrolysis of TGs and the release of free fatty acids into the circulation from adipose tissue. The serum TG rises and pancreatic lipase hydrolyzes the excess TGs producing large amounts of free fatty acids and free radicals, which injure acinar cells and cause pancreatic capillary ischemia [7]. The serum amylase levels might be spuriously low or they might be normal in $50 \%$ of hypertriglyceridemic pancreatitis patients at the time of admission or during their hospital course [8], as this case shows. This is because the hypertriglyceridemia interferes with the calorimetric reading of the assay [9]. Clinicians should rely on parameters other than the serum amylase and lipase levels to diagnose pancreatitis. Although the serum amylase and lipase and abdominal CT were not diagnostic, the lipidemic serum and high TG levels established the diagnosis of AP. The clinical course and routine management of hypertriglyceridemia-induced pancreatitis is similar to that of other causes. A thorough family history is important, as is the identification of secondary causes of hypertriglyceridemia, such as excess alcohol intake, thiazides, $\beta$-blockers, untreated type 2 diabetes mellitus, etc. A history of familial premature coronary vascular disease should be recorded. In this case, there was no family history of dyslipidemia. There was no history of other medications that can cause hypertriglyceridemia, suggesting that poorly controlled type 2 diabetes alone can lead to such high serum TG levels. Early hydration with a continuous insulin infusion resulted in rapid remission of not only the pancreatitis but also the lipid profile. The insulin infusion increases the peripheral synthesis of lipoprotein lipase contained in muscle and adipose tissue [10], and ongoing lipoprotein lipase function limits the degree of hypertriglyceridemia. However, the serum TG-lowering effect of insulin is not fully understood. To clarify this, further investigation of the mechanism of the increased TG in people with poorly controlled type 2 diabetes is required.

\section{REFERENCES}

1. Toskes PP. Hyperlipidemic pancreatitis. Gastroenterol Clin North Am 1990;19:783-91.

2. Anderson F, Thomson SR, Clarke DL, Buccimazza I. Dyslipidaemic pancreatitis clinical assessment and analysis of disease severity and outcomes. Pancreatology 2009;9:252-7.

3. Hegele RA. Monogenic dyslipidemias: window on determinants of plasma lipoprotein metabolism. Am J Hum Genet 2001;69:1161-77.

4. Lithell H, Vessby B, Walldius G, Carlson LA. Hypertriglyceridemia--acute pancreatitis--ischemic heart disease: a case study in a pair of monozygotic twins. Acta Med Scand 1987;221:311-6.

5. Solanki NS, Barreto SG, Saccone GT. Acute pancreatitis due to diabetes: the role of hyperglycaemia and insulin resistance. Pancreatology 2012; 12:234-9.

6. Kissebah AH, Vydelingum N, Murray R, Evans DJ, Hartz AJ, Kalkhoff $\mathrm{RK}$, et al. Relation of body fat distribution to metabolic complications of obesity. J Clin Endocrinol Metab 1982;54:254-60.

7. Mauriege P, Marette A, Atgie C, Bouchard C, Theriault G, Bukowiecki LK, et al. Regional variation in adipose tissue metabolism of severely obese premenopausal women. J Lipid Res 1995;36:672-84.

8. Yadav D, Pitchumoni CS. Issues in hyperlipidemic pancreatitis. J Clin Gastroenterol 2003;36:54-62.

9. Tsuang W, Navaneethan U, Ruiz L, Palascak JB, Gelrud A. Hypertriglyceridemic pancreatitis: presentation and management. Am J Gastroenterol 2009;104:984-91.

10. Sadur CN, Eckel RH. Insulin stimulation of adipose tissue lipoprotein lipase: use of the euglycemic clamp technique. J Clin Invest 1982;69:1119-25. 